\title{
Imaging Performance for Two Row-Column Arrays
}

Bouzari, Hamed; Engholm, Mathias; Nikolov, Svetoslav Ivanov; Stuart, Matthias Bo; Thomsen, Erik Vilain; Jensen, Jørgen Arendt

Published in:

IEEE Transactions on Ultrasonics, Ferroelectrics and Frequency Control

Link to article, DOI:

10.1109/TUFFC.2019.2914348

Publication date:

2019

Document Version

Peer reviewed version

Link back to DTU Orbit

Citation (APA):

Bouzari, H., Engholm, M., Nikolov, S. I., Stuart, M. B., Thomsen, E. V., \& Jensen, J. A. (2019). Imaging Performance for Two Row-Column Arrays. IEEE Transactions on Ultrasonics, Ferroelectrics and Frequency Control, 66(7), 1209-1221. https://doi.org/10.1109/TUFFC.2019.2914348

\section{General rights}

Copyright and moral rights for the publications made accessible in the public portal are retained by the authors and/or other copyright owners and it is a condition of accessing publications that users recognise and abide by the legal requirements associated with these rights.

- Users may download and print one copy of any publication from the public portal for the purpose of private study or research.

- You may not further distribute the material or use it for any profit-making activity or commercial gain

- You may freely distribute the URL identifying the publication in the public portal 


\title{
Imaging Performance for Two Row-Column Arrays
}

\author{
Hamed Bouzari, Mathias Engholm, Svetoslav Ivanov Nikolov, Matthias Bo Stuart, Erik Vilain Thomsen, and \\ Jørgen Arendt Jensen
}

\begin{abstract}
This study evaluates the volumetric imaging performance of two prototyped 62+62 row-column-addressed (RCA) 2-D array transducer probes using three Synthetic Aperture Imaging (SAI) emission sequences and two different beamsformers. The probes are fabricated using capacitive micromachined ultrasonic transducer (CMUT), and piezoelectric transducer (PZT) technology. Both have integrated apodization to reduce ghost echoes and are designed with similar acoustical features i.e., $3 \mathrm{MHz}$ center frequency, $\lambda / 2$-pitch, and $24.8 \times 24.8 \mathrm{~mm}^{2}$ active footprint. Raw RF data are obtained using an experimental research ultrasound scanner, SARUS. The SAI sequences are designed for imaging down to $14 \mathrm{~cm}$ at a volume rate of $88 \mathrm{~Hz}$. Two beamforming methods: Spatial matched filtering and rowcolumn adapted delay-and-sum are used for beamforming the RF data. The imaging quality is investigated through simulations and phantom measurements. Both probes on average have similar lateral full-width at half-maximum (FWHM) values, but the PZT probe has $20 \%$ smaller cystic resolution values and $70 \%$ larger contrast-to-noise ratio compared to the CMUT probe. The CMUT probe can penetrate down to $15 \mathrm{~cm}$, and the PZT probe down to $30 \mathrm{~cm}$. The CMUT probe has $17 \%$ smaller axial $\mathrm{FWHM}$ values. The matched filter focusing shows and improved B-mode image for measurements on a cyst phantom with an improved speckle pattern and better visualization of deeper lying cysts. The results of this study demonstrate the potentials of RCA 2-D arrays against fully addressed 2-D arrays, which are low channel count (e.g. 124 instead of 3,844), low acoustic intensity (MI $\leq 0.88$ and $\left.I_{\text {spta }} \leq 5.5 \mathrm{~mW} / \mathrm{cm}^{2}\right)$, and high penetration depth (down to $\left.30 \mathrm{~cm}\right)$, which makes 3-D imaging at high volume rates possible with equipment in the price range of conventional 2-D imaging.
\end{abstract}

\section{INTRODUCTION}

To obtain real time-resolved volumetric imaging with frame rates higher than $20 \mathrm{~Hz}, 2-\mathrm{D}$ transducer arrays are necessary [1], [2]. Such transducers were first developed in the early 1990s [3]. By placing the elements in a rectangular grid, the beam can be steered electronically in two perpendicular directions (azimuth and elevation) and hereby acquire data from a volume. To obtain an image quality similar to that of a 1-D transducer, the same number of elements in both lateral dimensions is needed. A 1-D array of 128 elements would translate into $128 \times 128=16,384$ elements in a 2-D matrix array. From a transducer fabrication perspective, fully addressing the matrix array elements poses

This work was financially supported by grant 82-2014-4 from the Danish Advanced Technology Foundation and from BK Ultrasound ApS (Herlev, Denmark).

H. Bouzari was with the Center for Fast Ultrasound Imaging, Department of Health Technology, Technical University of Denmark, 2800 Kgs. Lyngby, Denmark and is now with Fingerprint Cards AB.

M.B. Stuart, and J.A. Jensen* are with the Center for Fast Ultrasound Imaging, Department of Health Technology, Technical University of Denmark, 2800 Kgs. Lyngby, Denmark.

M. Engholm and E.V. Thomsen are with the Department of Health Technology, Technical University of Denmark, 2800 Kgs. Lyngby, Denmark.

S.I. Nikolov is with BK Medical, 2730 Herlev, Denmark.

${ }^{*}$ E-mail of corresponding author is: jaje@dtu.dk a great challenge for providing electrical connections to all the elements while maintaining a high element yield. The interconnecting wires between the 16,384 elements and the ultrasonic system result in a large, heavy cable, which excludes it from any practical use.

In 2003, Morton and Lockwood [4] suggested the rowcolumn-addressed (RCA) 2-D arrays as an alternative to fullyaddressed (FA) 2-D arrays. Row-column-addressing of 2-D arrays is a scheme to reduce the number of active channels needed for contacting the elements in the array. The idea is to contact the elements in the 2-D array either by their row or column index. Each row or column thereby acts as one large element. This effectively turns the array into two orthogonal 1-D arrays. The imaging principle relies on using one of the 1-D arrays as the transmit array. This creates a transmit field, which is a plane wave along the transmitting element height and a circular wave in orthogonal direction along the element width. The perpendicular 1-D array is used to receive, enabling receive focusing in the orthogonal dimension. The combination of transmit and receive focus provides focusing in a point in the volume, hence a volumetric image can be created. Whereas a $N \times N$ fully addressed array needs $N^{2}$ connections, a RCA array only needs $2 N$ connections. The RCA array can therefore have a larger aperture compared to the fully addressed array, having the same number of connections. A simulation study by Rasmussen and Jensen [5] and a measurements study [6], both compared the two different addressing schemes. With the same number of connections, a superior image quality is obtained using the RCA array due to its larger size.

An inherent drawback of the row-column-addressing, is that the long elements produce considerable edge effects, leading to ghost echoes in the beamformed image. Since the elements do not allow electronic control along their length, the ghost echoes cannot be removed with conventional electronic apodization. This issue was first addressed by Demoré et al. [7] and later investigated in detail by Rasmussen and Jensen [5]. Both studies concluded that integrating the apodization in the transducer itself was an effective way of solving the issue. Several ways of realizing the integrated apodization have been suggested, including a variable polarization of the piezo ceramic material [8] and varying the density of capacitive micromachined ultrasonic transducer (CMUT) cells [9].

Imaging with RCA 2-D arrays has been investigated based on simulations [4], [7], [10]-[12] as well as measurements with arrays fabricated in CMUT [13]-[17] and PZT [18]-[21] technologies separately. The purpose of this paper is to evaluate the imaging performance attainable for RCA 2-D arrays quantitatively and comparatively when employing synthetic aperture data acquisition and using two beamforming schemes developed specially for row-column imaging. The rectilinear 
volumetric imaging performance is investigated based on simulations and phantom measurements. The two probes are equipped with hardware static roll-off apodization connected to both ends of each row and column element [11], [17]. Both of the transducers are designed with similar acoustical features, i.e., dimensions, center frequency, and packaging, and plugged into the ultrasound research scanner, SARUS [22]. The detailed construction of the probes is described in [23].

A modified delay-and-sum (DAS) beamformer using the methods described in [11] is employed, where the size of the line elements are taken into account. The second beamformer uses the spatial matched filter (SMF) [24]-[26] methods for processing the RF data. Here the received signals are modeled in Field II and the signal shape is used as a spatial matched filter in the beamformation [24]. Three different SAI [27] sequences were designed for imaging down to $14 \mathrm{~cm}$ for a volume rate of $88 \mathrm{~Hz}$. The first sequence uses 62 virtual lines sources behind the array, the second sequence utilizes 62 single element transmissions, and the last sequences employes lines sources focused in front of the arrays. The returned echoes are collected with all column elements, focused with the two beamformers for all emissions, and then combine coherently for the full rectilinear volume. The quality of the B-mode volumes acquired with both probes, i.e., spatial resolution, contrast resolution, and SNR was determined based on simulations and measurements on several phantoms.

Initial results of this study have been published as conference papers [28]-[30] for the PZT and CMUT technologies using a SAI sequence with single element transmission. This study extends the DAS and SMF beamformation methods for the focused and defocused SAI sequences as well as reporting on the resolution, probe temperature, and acoustic intensities. Furthermore, the focusing ability of RCA 2-D arrays are compared with fully addressed 2-D arrays.

\section{IMAGING WITH RCA AND 2-D ARRAYS}

This Section gives an overview of the benefits of using rowcolumn arrays in terms of resolution and penetration depth coming from the increased size compared to full matrix arrays. The challenges in terms of contrast are also addressed, and ways to mitigate this by using synthetic aperture imaging is described and investigated in the remaining part of the paper.

Principally, the achievable lateral resolution of an ultrasound system is defined by its two-way beam width at the focal depth using conventional focusing on both reception and transmission [31]. However, in imaging with an RCA 2-D array, the focusing in the transmit direction is independent from the receive direction, thus, the spatial resolution in each direction can differ from the other direction depending on how well the focus lines are generated in each direction. Due to the perpendicular orientation of the transmit and receive apertures in RCA 2-D arrays, only one-way focusing is possible in each lateral direction [7], [11], [26], and this should be taken into account when designing the imaging sequences.

The Fresnel approximation states that the pressure field at the focus may be described by the Fourier transform of the transducer aperture. A finite array of transducer elements has an

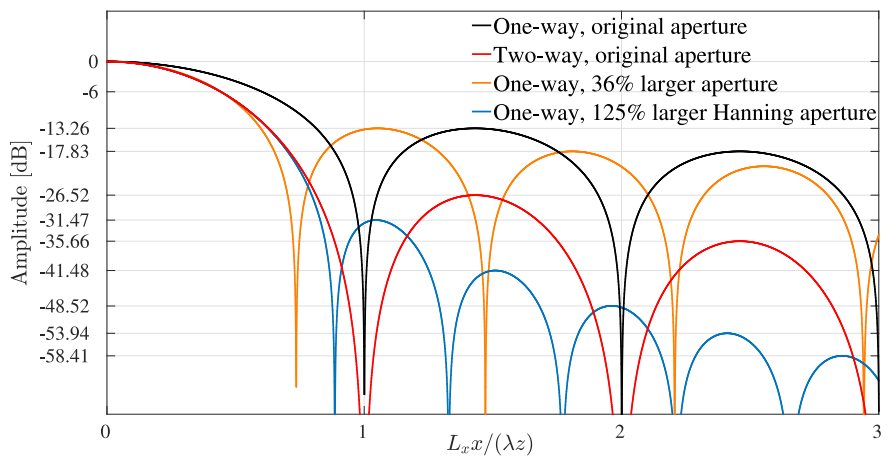

Fig. 1. Resulting $\mathrm{CW}$ field at the focal distance from a one-way focused array (black), a one-way focused array with $36 \%$ larger aperture side-length (orange), and a two-way focused array (red). The two former are plotted using a normalized (1), while the latter uses (3). The lateral profile of a one-way focused array with $125 \%$ larger aperture side-length with Hanning apodization is shown in blue.

aperture $A$, described by a simple rectangular window function along one lateral dimension, where the Fourier transform is a sinc function. Denoting the size of this array along the $x$ dimension $L_{x}$, the position along the array $x$ ( $x=0$ being the center of the array), the wavelength of the ultrasound wave $\lambda$, and the mass density of the medium $\rho_{a}$, the continuous wave (CW) pressure field at depth $z$ becomes [31]:

$$
p_{x, \text { one-way }}=\mathfrak{F}[A]=\frac{L_{x} \sqrt{\rho_{a}}}{\sqrt{\lambda z}} \operatorname{sinc}\left(\frac{L_{x} x}{\lambda z}\right),
$$

where $\mathfrak{F}$ denotes the Fourier transform. It is assumed that $z$ is at the transducer focus. The full-width at half-maximum (FWHM) of the sinc function is

$$
\mathrm{FWHM}_{\text {one-way }}=\frac{1.208 \lambda z}{L_{x}}=1.208 \lambda f_{\#} .
$$

The FWHM resolution for a given wavelength and depth, thus, scales with the array size. The subscript "one-way" is to emphasize that the FWHM is for focusing of only the transmit aperture (or only the receive aperture due to acoustic reciprocity). An RCA array can only perform one-way focusing in each lateral dimension, if conventional DAS beamforming is used, and its detail resolution is therefore defined by (2). As opposed to this, a 2-D matrix array can focus in each lateral dimension both in transmit and receive. The resulting pulse-echo field is proportional to the Fourier transform of the convolved transmit and receive apertures [32]. If the same aperture is used for transmitting and receiving, the pulse-echo field along one dimension becomes:

$$
p_{x, \text { two-way }} \propto \mathfrak{F}\left[\begin{array}{c}
* \\
*
\end{array} A\right]=\mathfrak{F}[A] \mathfrak{F}[A]=(\mathfrak{F}[A])^{2} .
$$

The FWHM for two-way focusing is:

$$
\mathrm{FWHM}_{\text {two-way }}=\frac{0.886 \lambda z}{L_{x}}=0.886 \lambda f_{\#} .
$$

and the ratio between one-way and two-way focusing is:

$$
\frac{\text { FWHM }_{\text {one-way }}}{\text { FWHM }_{\text {two-way }}}=\frac{1.208 \lambda f_{\#}}{0.886 \lambda f_{\#}} \simeq 1.36 \text {. }
$$

Thus, for the same aperture size, the theoretically expected FWHM of an RCA array is $36 \%$ larger than the FWHM of a 
two-way focused 2-D array. Based on the FWHM, the detail resolution for the two types of arrays will consequently be equal, if the side-length of the RCA array is increased by $36 \%$ relative to the fully addressed 2-D array. In Fig. 1, the resulting fields from the one-way focused array (black), the two-way focused array (red), and the one-way focused array with a $36 \%$ larger side-length (orange) are plotted. It is seen that the FWHM of the two latter are indeed identical.

The ratio between the number of elements in an RCA array and a fully addressed 2-D matrix array with equal detail resolution is then:

$$
\frac{\text { No. elem. in fully addr. array }}{\text { No. elem. in RCA array }}=\frac{N^{2}}{1.36 \cdot 2 \cdot N}=\frac{N}{2.72} .
$$

To attain the same lateral resolution for both fully addressed and RCA 2-D arrays, the number of row or column elements on an RCA array has to get increased only by a factor of $1.208 / 0.886=1.36$, i.e., by a factor of $2 \cdot 1.36=2.72$ for the total number of elements. For instance for a 2-D array with $256 \times 256$ elements, row-column addressing corresponds to a reduction in the total number of channels by $99.6 \%$, i.e., from 65,536 channels to 512 channels. Any $N+N$ channel RCA array with $N \geq 3$ will, thus, achieve a better detail resolution than a fully addressed 2-D array with the same total number of channels.

Due to the low channel count of the RCA 2-D arrays, it is possible to fabricate 2-D arrays with a larger aperture size, which can yield an increased penetration depth. The benefits in FWHM resolution and array area is visualized in Fig. 2, where the two array types also have been compared to a Mills cross array [3], [33]. They contain a single line of array elements along the two diagonals of a fully populated matrix array. The Mills cross array has a slightly better resolution, but a considerably smaller surface area, which translates to a lower emitted pressure and lower receive sensitivity. For a 1024 channel RCA array the surface area is more than 2 orders of magnitude larger than for the fully populated array. For the 124 channels employed in this paper, the fully populated array is so small and with such few elements that imaging is hardly possible.

However, changing the aperture size will only affect the argument in the sinc function in (1), not the shape of the function, and the side-lobe levels might be high for the RCA array. This can be mitigated by employing apodization on both the transmit and receive elements. This will lower the effective size of the array, which can be compensated for by increasing the number of elements. An example is shown in Fig. 1, where an array with $125 \%$ increased size attains lower side-lobes than for two-way focusing. The increase in number of elements per side is then 2.25 , so any $N+N$ channel RCA array with $N \geq 5$ potentially outperforms fully populated arrays. Further, employing synthetic aperture imaging makes it possible to combine many emissions coherently to increase the main lobe and relatively lower the side lobes, if the beamforming is performed as described in Section V. The resolution and contrast can be maintained at the optimal value through depth by employing the synthetic aperture imaging described in Section III.
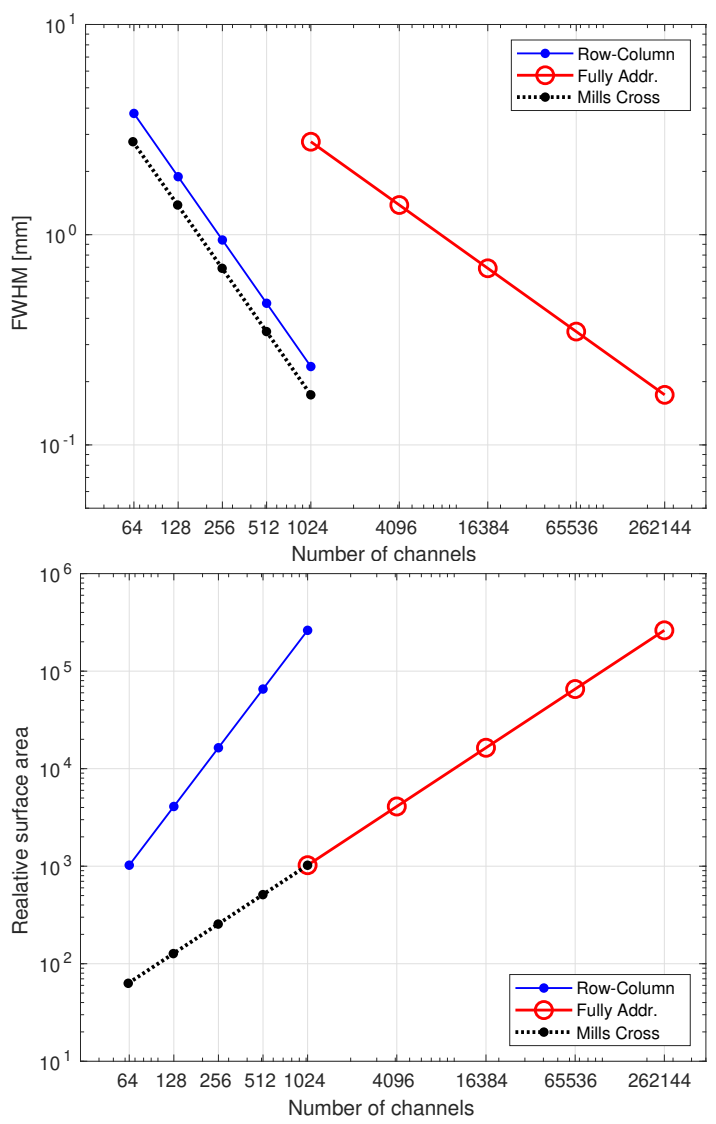

Fig. 2. Comparison of resolution (top) and array size (bottom) between a RCA array, a fully populated array and the Mills cross array.

The simple equations above only give a rough estimate of the resolution, contrast, and penetration, and either broad-band simulations or measurements have to be employed to determine the true performance. These are revealed for the specific arrays in subsequent Sections.

\section{SAI IMAGING}

Two different SAI [27] sequences were designed for imaging down to $14 \mathrm{~cm}$. The first sequence utilizes 62 virtual line sources behind the array by adjusting the transmit delays of all the row elements. A virtual line source emits cylindrical pressure waves, which propagate as plane waves in one lateral dimension and as arcs in the perpendicular lateral dimension. The second sequence utilizes 62 single element transmissions on the row elements. In both sequences the echoes are collected with all the column elements. For a speed of sound of $1540 \mathrm{~m} / \mathrm{s}$, $182 \mu \mathrm{s}$ is required to acquire data from a single emission to a depth of $14 \mathrm{~cm}$ corresponding to a volume rate of $88 \mathrm{~Hz}$.

RF data are used for beamforming a low-resolution volume for every emission and finally, by summing all the lowresolution volumes, a high-resolution volume is generated.

\section{Defocused SAI Sequence Choice of Parameters}

To accomplish the best performance, the location and number of virtual sources have to be optimized in a trade-off between spatial resolution, field-of-view, and signal-to-noise ratio (SNR). 


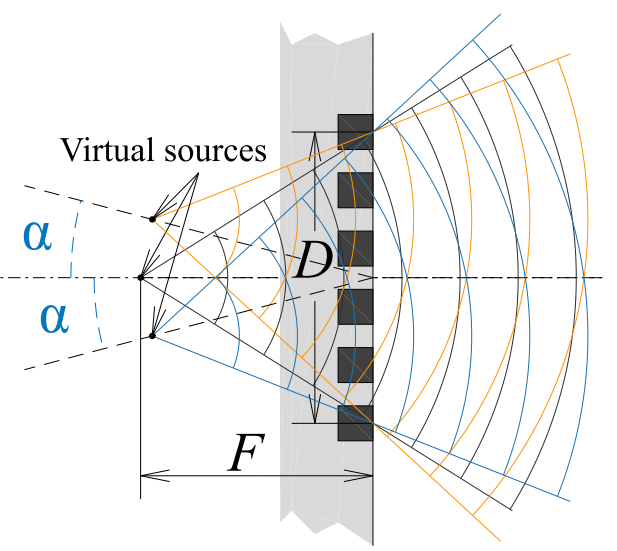

Fig. 3. SAI sequence with beam steering and translation of virtual line sources The figure shows three virtual line sources located behind the array. $D$ is the active aperture, $\alpha$ is the maximum steering angle, and $F$ denotes the distance to the active aperture centre, i.e., $f_{\#}=F / D$.
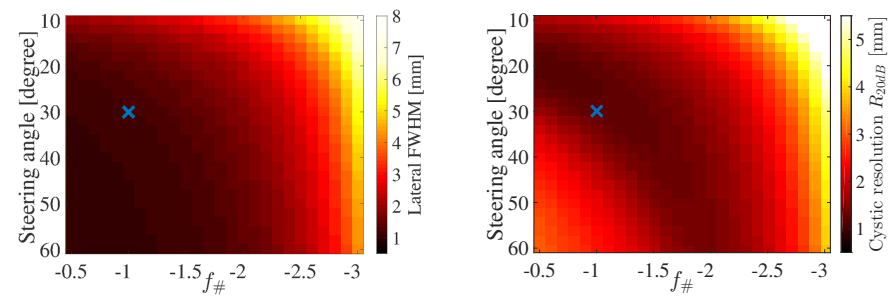

Fig. 4. Lateral FWHM and CR as function of steering angle and transmit $f_{\#}$ for a point target located along the central axis at depth of $20 \mathrm{~mm}$. The simulated RF data are beamformed with the DAS method. As a trade-off between contrast and spatial resolution, a steering angle of $\pm 30^{\circ}$ and a transmit $f_{\#}=-1$ (indicated with a blue marker) are chosen for the defocused SAI sequence.

Fig. 3 illustrates the position of three virtual line sources behind the array. In the figure, $D$ is the active aperture, $\alpha$ is the maximum steering angle, and $F$ denotes the distance to the active aperture centre, i.e., $f_{\#}=F / D$. The rest of the virtual line sources are inter-spaced equally between the two virtual line sources placed at the edges. A simulation parameter study is carried out over the maximum steering angle for placing the 62 virtual line sources and over the transmit $f_{\#}$ of the defocused SAI sequence to image a point scatterer at a depth of $20 \mathrm{~mm}$ in front of the array. The lateral FWHM and contrast ratio (CR) values of the beamformed point spread function (PSF)s for maximum steering angles in range of $\pm 10^{\circ}$ to $\pm 60^{\circ}$ and transmit $f_{\#} \mathrm{~s}$ from -3 to -0.5 are shown in Fig. 4 . The criteria to choose the best parameters is to have the best contrast and spatial resolution for the lowest steering angle and transmit

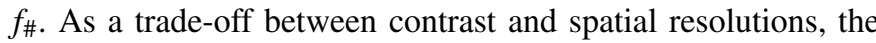
maximum steering angle of $\pm 30^{\circ}$ and transmit $f_{\#}=-1$ are chosen for the defocused SAI sequence. The parameters of both SAI sequences are listed in Table II.

\section{ARrAy DESIGN AND FABRICATION}

The general design of the RCA arrays is based on the findings by Rasmussen et al. [11] and Christiansen et al. [17]. The arrays consist of 62 row elements and 62 column elements, and four apodization regions. Only the $62+62$ elements are connected
Table I

TRANSDUCER DIMENSIONAL PARAMETERS

\begin{tabular}{lccl}
\hline Parameter & CMUT & PZT & Unit \\
\hline Number of elements & $62+62$ & $62+62$ & - \\
Number of apodization region electrodes & 4 & 4 & - \\
Element pitch & 270 & 270 & $\mu \mathrm{m}$ \\
Element width & 265 & 245 & $\mu \mathrm{m}$ \\
Kerf & 5 & 25 & $\mu \mathrm{m}$ \\
Element length & 24.84 & 24.84 & $\mathrm{~mm}$ \\
Length of apodization regions & 4.05 & 4.05 & $\mathrm{~mm}$ \\
Array outer dimensions (square) & 26.3 & 26.3 & $\mathrm{~mm}$ \\
\hline
\end{tabular}

to beamformer channels. The design of the RCA array can therefore be divided into two parts: The central region and the apodization region.

The central part of the array may be considered as a conventional RCA array. The top and bottom electrodes are placed orthogonal to each other. Between the top and bottom electrodes is the "active" material, which is either the CMUTs or the piezoelectric material. The element contacts are placed alternately on each side of the array. The top elements can be used as a 1-D array by grounding all of the bottom elements, and the bottom elements can be used as an orthogonal 1-D array by grounding all of the top elements.

The four apodization regions are located outside the central part of the array and are added to avoid the abrupt truncation of the elements, which gives rise to the ghost echoes [11]. The apodization regions are placed on each side of the central region, and the apodization values follow a Hann function from the edge of the central part to the edge of the array, where the apodization is 0 . The apodization was originally developed for the CMUT array [17], but has been adapted to have the same dimension and roll-off characteristic for the PZT array.

Two arrays are fabricated using the design introduced above: one based on CMUT technology and one based on piezoelectric transducer (PZT) technology. The pitch, number of elements, active footprint, center frequency, and excitation voltage are designed to be identical for the two arrays. This makes it possible to evaluate and compare the row-column-addressing scheme based on two different technologies. A center frequency of $3 \mathrm{MHz}$ was chosen with lambda half pitch and an excitation voltage of $\pm 75 \mathrm{Vac}$. The dimensional parameters of both arrays are given in Table I.

The fabrication and characterization of the arrays have been thoroughly described in [23].

\section{BEAMFORMING AND ACQUiSITION METHODS}

In the SAI technique, both DAS and SMF beamforming methods have been used for generating the low-resolution B-mode volumes before in phase summation of them to produce a higher resolution B-mode volume. The adaptation of these beamformation methods to RCA 2-D arrays, the quality assessment measures of the B-mode volumes, and the SAI $\mathrm{B}$-mode imaging sequences are explained in this Section.

\section{A. DAS Beamforming}

The DAS beamforming method for RCA 2-D arrays was presented in [11]. This method models each row or column 
element as a line-segment, and therefore calculates the delays based on the shortest geometrical distance between an imaging point and this line-segment. The delayed signals are then summed coherently across elements and transmit emissions to generate a high resolution image with low side-lobes. An expanding aperture for both transmissions and receiving elements with an $f \#$ of 1 and a Hanning apodization was employed.

\section{B. SMF Beamforming}

Normal DAS focusing assumes that the spatial impulse response of the transducer is a delta function, and that the alignment can be performed by merely delaying the responses. This is appropriate in the far-field for small element arrays and at the focus for single element transducers. However, in the near-field, the pulse-echo spatial impulse responses are different from a delta function [24].

As an alternative to dynamic receive focusing using DAS beamforming, the signal from each channel of an array can be spatially matched filtered to align its output with that from the other channels [24]-[26], [29]. This was suggested by Yen [26] for use on RCA arrays. In this work, Field II Pro [34]-[36] is used for calculations of the SMF coefficients, based on the arrays dimensions, excitation pulse, and the measured impulse response.

\section{Imaging Quality Assessment Measures}

The imaging performance is computed in terms of SNR, FWHM, CR [37]-[39], and contrast-to-noise ratio (CNR).

Table II

SETUP CONFIGURATION FOR THE SIMULATIONS AND MEASUREMENTS

\begin{tabular}{lllc}
\hline SAI sequences & Single element & $f_{\#}=-1$ & \\
\hline Pulse repetition frequency & 5 & 5 & $\mathrm{kHz}$ \\
No. of active elements in Tx & 1 & 62 & - \\
Scan depth (max range) & 14 & 14 & $\mathrm{~cm}$ \\
Emission center frequency & 3 & 3 & $\mathrm{MHz}$ \\
Sinusoid emission cycles & 2 & 2 & - \\
Focus in transmit & 0 & -16.8 & $\mathrm{~mm}$ \\
Focus in receive & Dynamic & Dynamic & - \\
Tx electronic apodization & - & Hann. & - \\
Rx electronic apodization & Rect. & Rect. & - \\
Sampling frequency & 70 & 70 & $\mathrm{MHz}$ \\
Tx voltage for PZT \& CMUT & \pm 75 & \pm 75 & $\mathrm{~V}$ \\
DC bias voltage for CMUT & 190 & 190 & $\mathrm{~V}$ \\
\hline
\end{tabular}

\section{Equipment and Measurement Setup}

The dimensional parameters of both arrays are found in the paper describing the transducers [23]. The probes are plugged into the experimental ultrasound scanner, SARUS [22]. The measured RF signals are beamformed using a MATLAB (MathWorks Inc., Massachusetts, USA) implementations of the DAS and SMF beamformers.
To evaluate the imaging performance of both probes, several ultrasound phantoms are used. An iron needle with diameter of $300 \mu \mathrm{m}$ facing towards the transducer along its central axis, was used as a point target in a water bath for characterizing the 3-D PSF. To evaluate the FWHM and the CR as a function of depth, a geometrical copper wire phantom was used as line targets, where wires were located at different depths with $1 \mathrm{~cm}$ spacing. The wire grid phantom has three columns separated by $1 \mathrm{~cm}$ and each has 13 rows of wires.

A tissue mimicking phantom with cylindrical anechoic targets, model 571 from Danish Phantom Design (Frederikssund, Denmark) with attenuation of $0.5 \mathrm{~dB} /(\mathrm{cm} \mathrm{MHz})$ was used for SNR and contrast measurements.

The mean impulse responses for the probes have been reported in [23], [28], [30], however the phase delay of the individual elements was found by cross-correlating the impulse response for each element with the mean impulse response and interpolating to find the lag of the maximum of the crosscorrelation. The phase delay was then calculated by dividing the time it takes the wave to travel one wavelength at $3 \mathrm{MHz}$ and multiplying it by $360^{\circ}$, to obtain the phase delay in degrees.

No curvature is seen of the CMUT, however the PZT is observed to curve. The bottom/column elements phase delays are seen to have a concave profile, whereas the top/row elements have a convex profile. This saddle shape is believed to originate from stress build up during the assembly.

\section{IMAGING PERFORMANCE ASSESSMENT}

Fig. 5 illustrates three cross-planes (azimuth, elevation, and C-plane) of the volumetric pulse-echo beam patterns measured with both probes in comparison with Field II simulations using the DAS beamformation method. The iron needle faces towards the transducer, and it is imaged with the single element transmissions SAI sequence. A Hanning apodization is applied over the receive and synthesized transmit apertures. The measured pulse-echo impulse responses of both probes [23], the curvature of the PZT probe as well as the diameter of the needle are taken into account for the simulations by imaging a disk consisting of 500 point targets to represent the tip of the needle.

The simulated PZT PSFs are slightly asymmetric due to the curvature of this probe, and this is also seen in the measured PSFs. Note also the secondary lobes after the main lobe along the axial direction for both simulated PSFs of the probes at the range of $20 \mathrm{~mm}$ to $22 \mathrm{~mm}$ of depth, which are due to the internal reflections from the RF shielding foils covering the arrays. These secondary reflections are visible in the impulse responses of the probes [23]. Among these secondary echoes are the edge echoes, which originate from the either ends of the line elements. They were not fully suppressed below $40 \mathrm{~dB}$ [11], [17] due to imperfect static roll-off apodization. The way to classify them is only based on the timing of their occurrences as explained in the paper [23]. The same RF data are used for the SMF beamforming method. FWHM and the CR of the simulated and measured 3-D PSFs are listed in Table III for both DAS and SMF beamforming methods. For comparison, a simulated and DAS beamformed PSF of a fully 


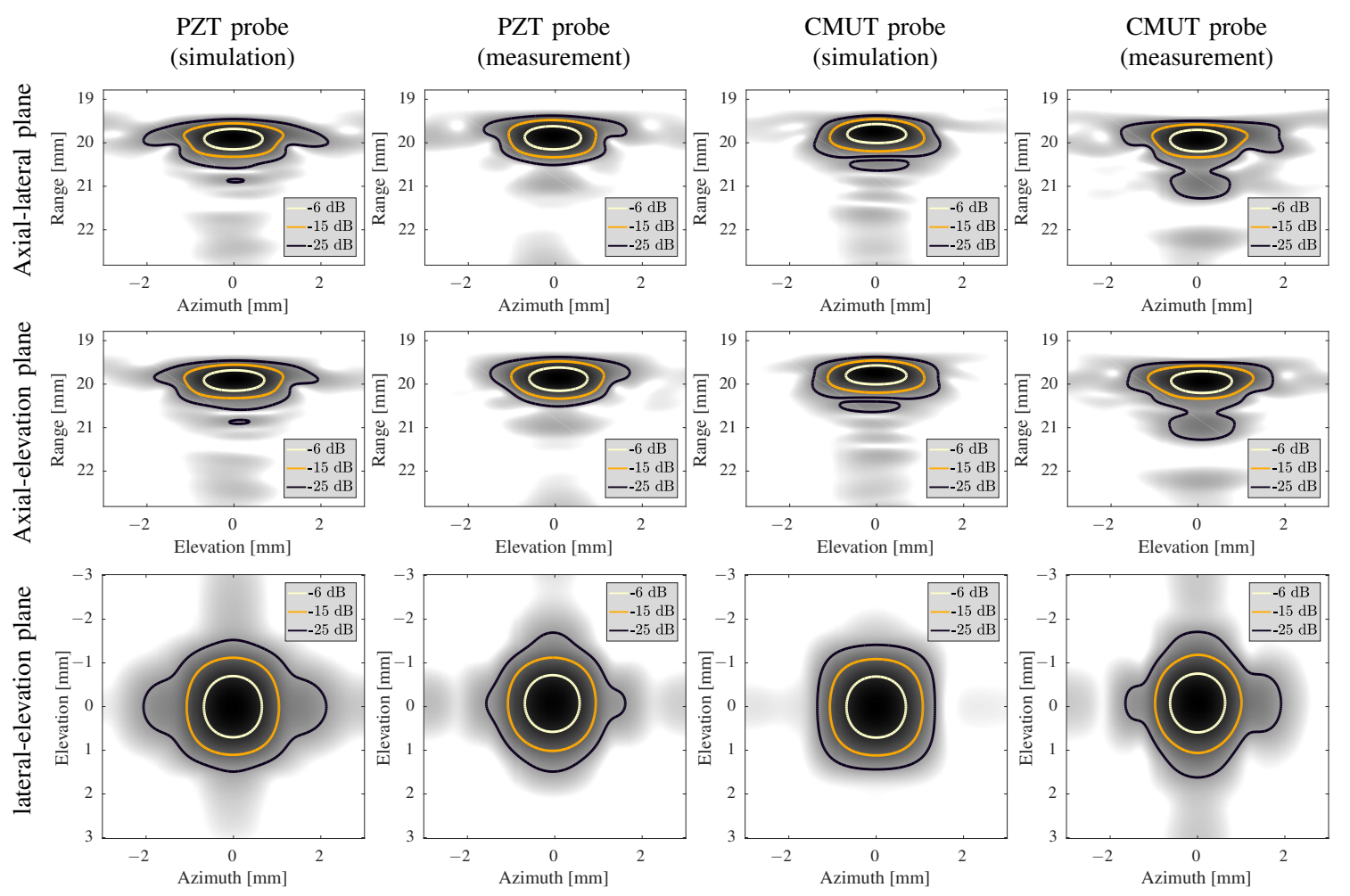

Fig. 5. Comparison of simulated and measured PSFs using DAS beamformation and Hanning electronic apodization. Three cross-planes (azimuth, elevation, and C-plane) are shown from a volume of $6 \times 6 \times 4 \mathrm{~mm}^{3}$ at a dynamic range of $40 \mathrm{~dB}$. The origin corresponds to the center of the transducer surface aligned with a point target (an iron needle) positioned at $(0,0,19.8) \mathrm{mm}$. Data were acquired with the PZT and CMUT probes using SAI with 62 single element emission events. The calculated FWHM and CR are listed in Table III.

addressed $62 \times 62$ (i.e. 3,844 elements) $3 \mathrm{MHz} \lambda / 2$-pitch 2-D array is also provided in Table III. The 3-D PSF was imaged using a SAI sequence with $32 \times 32$ virtual point sources behind the array, corresponding to 1,024 emissions. The maximum steering angle of each virtual point source is equally distributed between $\pm 30^{\circ}$ in each lateral direction, i.e., a $60^{\circ} \times 60^{\circ}$ fieldof-view with a transmit $f_{\#}=-1$.

Due to the similar dimensions of the probes, simulations for both arrays show symmetric patterns in the C-plane. Measurements with the PZT probe show symmetry at $-6,-15$, and $-25 \mathrm{~dB}$, however measurements with the CMUT probe show wider elevation and narrower azimuth patterns. This is related to the sensitivity differences between rows and columns in the CMUT probe as described in [23]. The axial FWHM values of the CMUT probe are $17 \%$ smaller than for the PZT probe due to its higher bandwidth. This also causes the CMUT probe to have larger CR values compared to the PZT probe. Although both DAS and SMF beamformation techniques have resulted in similar lateral and elevation FWHM values, CR values have improved using the SMF method. During SMF beamforming with broadband RF signals, a 2-D spatiotemporal matched filtering is applied, which involves not only a lateral convolution in space but also a temporal convolution in the axial direction. The temporal convolution elongates the pulse-echo response, which results in a worse axial spatial resolution, consequently leading to a larger speckle size in the axial direction.

The more pronounced secondary lobes in the measurements compared with the simulations in azimuth and elevation planes
Table III

FWHM AND CR OF SIMULATED AND MEASURED 3-D PSFS

\begin{tabular}{|c|c|c|c|c|c|c|c|c|}
\hline & \multicolumn{2}{|c|}{ Simulation } & \multicolumn{2}{|c|}{ Measurement } & \multirow[b]{2}{*}{ FA } & \\
\hline & & & CMUT & PZT & CMUT & PZT & & \\
\hline \multirow{7}{*}{ 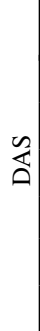 } & & $R_{6 \mathrm{~dB}}$ & 0.37 & 0.35 & 0.55 & 0.42 & 0.27 & $\mathrm{~mm}$ \\
\hline & ્ & $R_{12 \mathrm{~dB}}$ & 1.03 & 0.86 & 1.15 & 0.85 & 0.98 & $\mathrm{~mm}$ \\
\hline & & $R_{20 \mathrm{~dB}}$ & 3.06 & 2.9 & 3.32 & 2.75 & 1.27 & $\mathrm{~mm}$ \\
\hline & & $R_{35 \mathrm{~dB}}$ & 4.17 & 4.01 & 4.54 & 4.43 & 3.9 & $\mathrm{~mm}$ \\
\hline & & Axial & 0.44 & 0.45 & 0.36 & 0.4 & 0.37 & $\mathrm{~mm}$ \\
\hline & 进 & Azimuth & 0.77 & 0.8 & 0.93 & 0.92 & 0.51 & $\mathrm{~mm}$ \\
\hline & 㞋 & Elevation & 0.78 & 0.8 & 0.88 & 0.88 & 0.51 & $\mathrm{~mm}$ \\
\hline \multirow{7}{*}{$\sum_{n=1}^{1}$} & \multirow{4}{*}{ Û } & $R_{6 \mathrm{~dB}}$ & 0.37 & 0.36 & 0.45 & 0.42 & - & $\mathrm{mm}$ \\
\hline & & $R_{12 \mathrm{~dB}}$ & 0.7 & 0.64 & 1.15 & 0.81 & - & $\mathrm{mm}$ \\
\hline & & $R_{20 \mathrm{~dB}}$ & 2.7 & 2.52 & 3.3 & 2.5 & - & $\mathrm{mm}$ \\
\hline & & $R_{35 \mathrm{~dB}}$ & 3.7 & 3.61 & 4.13 & 4 & - & $\mathrm{mm}$ \\
\hline & \multirow{3}{*}{$\sum_{i}^{\sum_{i}}$} & Axial & 0.5 & 0.52 & 3.3 & 2.5 & - & $\mathrm{mm}$ \\
\hline & & Azimuth & 0.83 & 0.82 & 0.97 & 0.96 & - & $\mathrm{mm}$ \\
\hline & & Elevation & 0.84 & 0.83 & 0.92 & 0.91 & - & $\mathrm{mm}$ \\
\hline
\end{tabular}

of Fig. 5 are due to the reflections coming towards the transducer from the shaft of the needle. Thereby, the transmitted waves have no deconstructive effect after the tip of the needle, which is not the case in simulations for an ideal point target. This had a more dramatic effect on the SMF beamforming method, which resulted in a larger axial FWHM reported in 


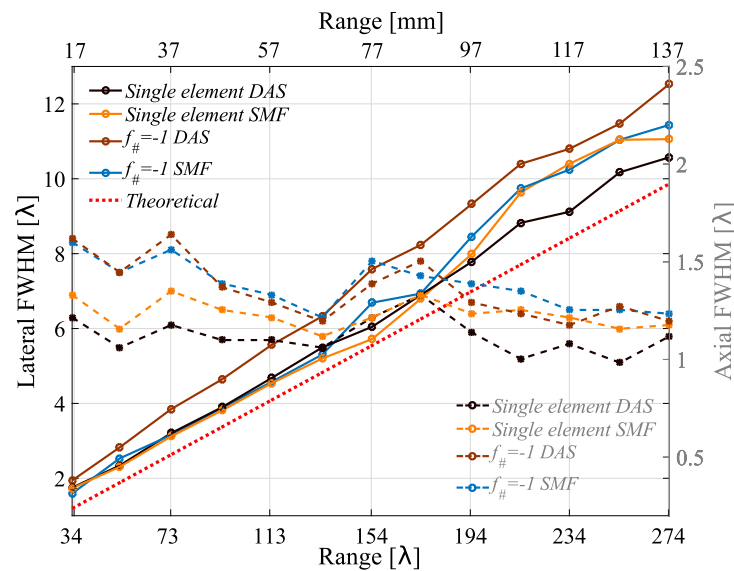

(a) PZT probe

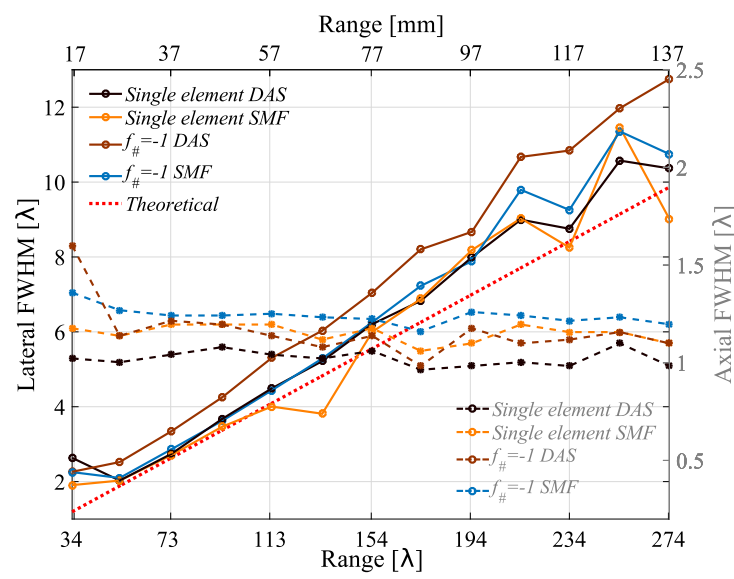

(b) CMUT probe

Fig. 6. Axial and lateral FWHM of the PZT (a) and CMUT (b) probes as a function of depth for DAS beamformed images of the wire phantom. The solid lines correspond to lateral FWHM (left axis) and the dashed lines correspond to axial FWHM (right axis). $\lambda$ was calculated for soft tissue and is $0.5 \mathrm{~mm}$. The red dotted line shows the estimated lateral FWHM based on the Fresnel approximation in (2).

\section{Table III.}

To study the focusing abilities of both probes as a function of depth, a wire grid phantom is used to quantify the line spread function (LSF) characteristics of both probes, since the echoed signals from the needle are too low at higher depths. The diameter of the wires is $200 \mu \mathrm{m}$, which is smaller than a wavelength in water, and therefore it is used as line targets. The phantom has three columns of wire, which are separated by $10 \mathrm{~mm}$ in the axial and lateral directions. Both of the SAI sequences are used to image the wire phantom with the PZT and CMUT probes by placing them centered around the middle column. A volume region of $26 \times 10 \times 5 \mathrm{~mm}^{3}$ centered around each beamformed wire in the middle column is used for the LSF characteristics evaluation as a function of depth. Fig. $6 a$ and Fig. 6b illustrate the calculated FWHM in the lateral and axial directions for both of the SAI sequences with the PZT and CMUT probes at different depths. Using the SAI sequence with transmit $f_{\#}=-1$ has increased the axial FWHM values of the PZT probe compared with the SAI sequence with single element at a time. This is because of the phase delay differences between the PZT elements, which have been reported in [23].

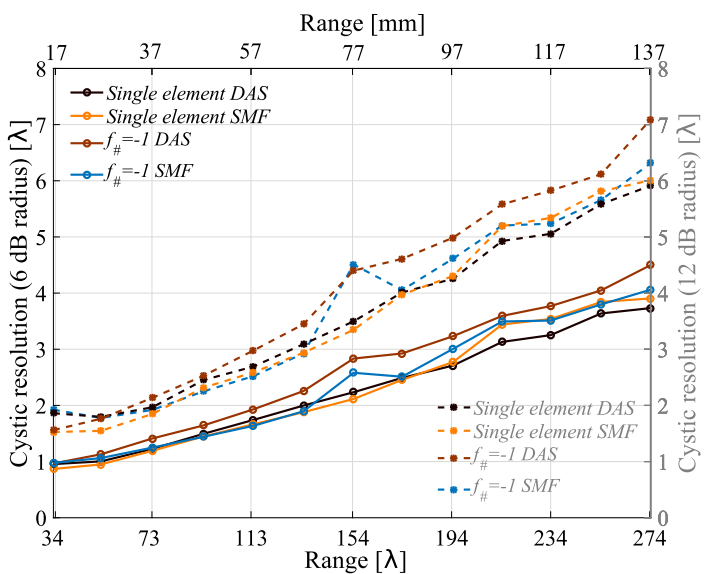

(a) PZT probe

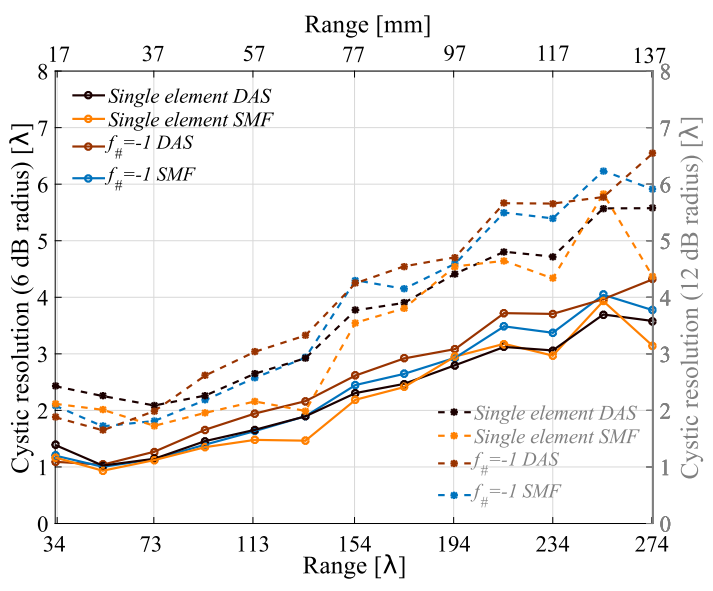

(b) CMUT probe

Fig. 7. Cystic resolution for $R_{6 \mathrm{~dB}}$ and $R_{12 \mathrm{~dB}}$ radius of the PZT (a) and CMUT (b) probes as a function of depth. Calculated over beamformed images of the wire phantom. The solid lines correspond to $R_{6 \mathrm{~dB}}$ (left axis) and the dashed lines correspond to $R_{12 \mathrm{~dB}}$ (right axis). $\lambda$ for soft tissue is $0.5 \mathrm{~mm}$.

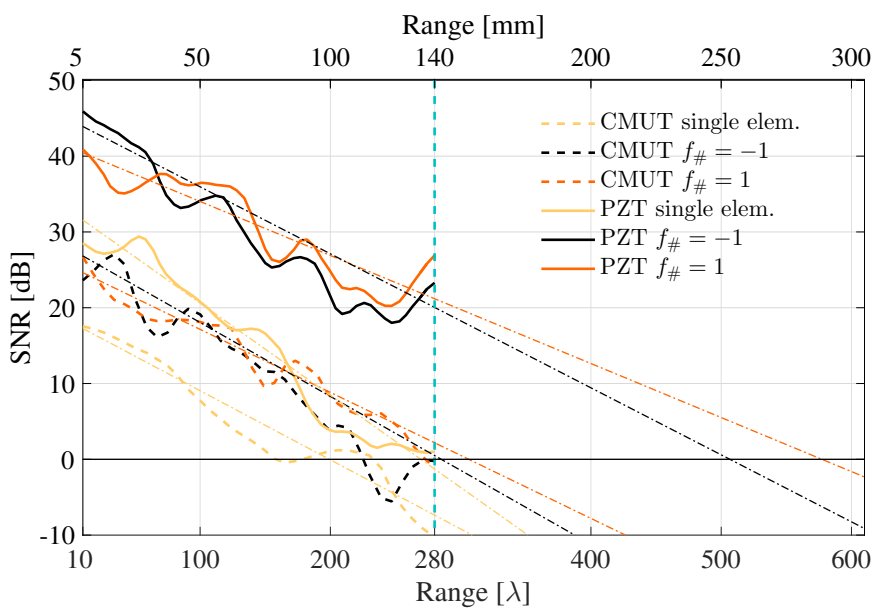

Fig. 8. SNR of the PZT and the CMUT probes on a region of a tissue mimicking phantom with no cyst and acoustical attenuation of $0.5 \mathrm{~dB} /(\mathrm{cm} \mathrm{MHz})$. The dash-dotted lines are linearly fitted to each curve. $\lambda$ for soft tissue is $0.5 \mathrm{~mm}$. The blue line indicates the maximum depth of the measured data

Likewise what was observed previously with the 3-D PSFs, here for the LSFs, the CMUT probe has smaller FWHM values in the axial direction comparing with the PZT probe. Although 

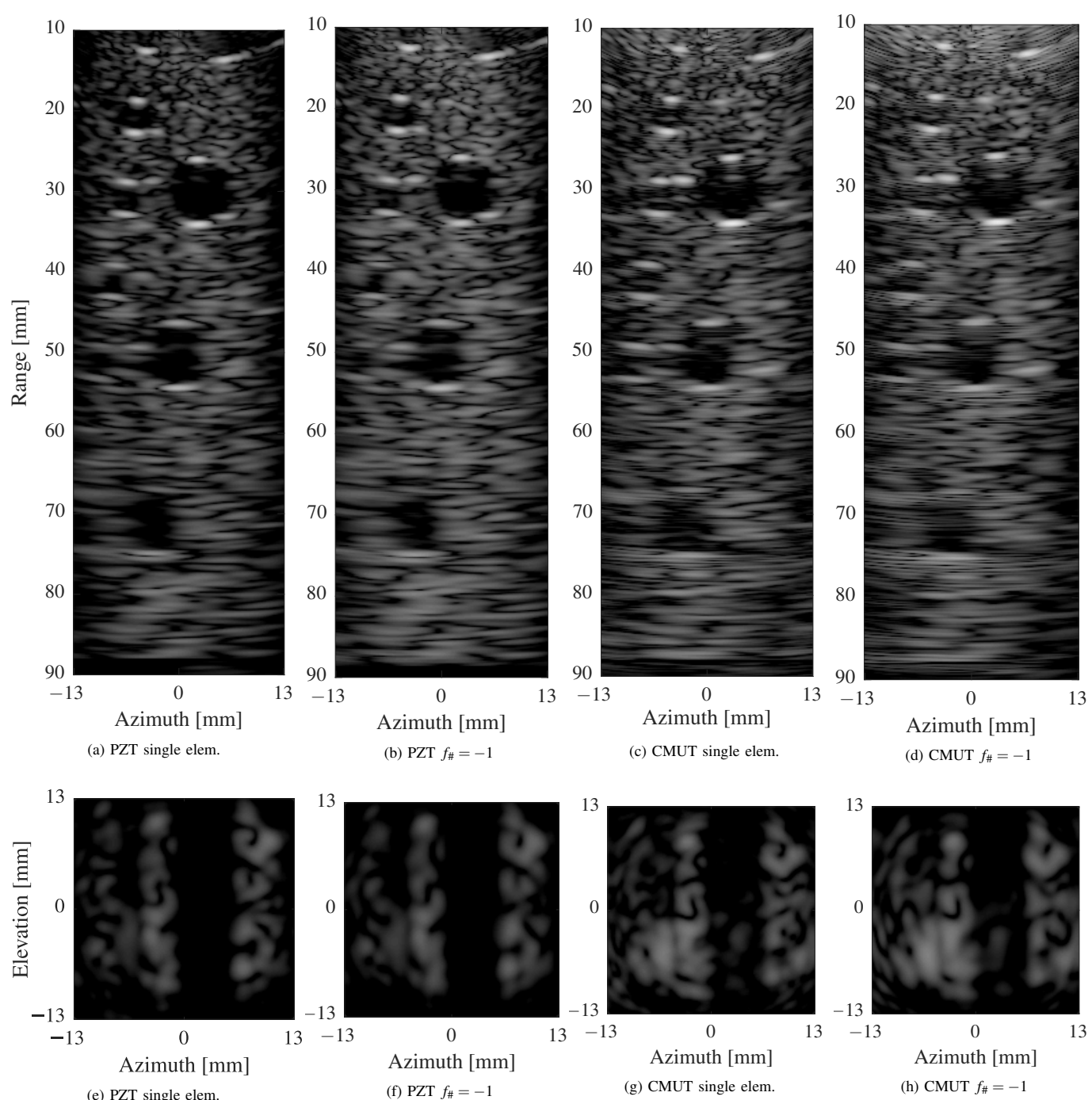

Fig. 9. Volumetric imaging of a tissue mimicking phantom using both PZT and CMUT probes and beamformed with the DAS method. Two cross-planes (azimuth and C-plane) are shown from a volume of $26 \times 26 \times 85 \mathrm{~mm}^{3}$ at a dynamic range of $40 \mathrm{~dB}$. Data were acquired with the PZT probe using the SAI sequence with 62 single element emissions (PZT: (a) and (e)), (CMUT: (c) and (g)), and also using the SAI sequence with transmit $f_{\#}=-1$ (PZT: (b) and (f)), (CMUT: (d) and (h)). The C-planes are at the depth of $30 \mathrm{~mm}$. For the full-sized volumes of the SAI sequence with transmit $f_{\#}=-1$ see the videos in the supplementary materials.

the lateral FWHM values increase linearly with depth similar to (2), the SMF beamforming has lowered the FWHM values for the SAI sequence with transmit $f_{\#}=-1$. As it is illustrated in Fig. 7a and Fig. 7b, CR values increase almost linearly with depth for both probes.

A volume region of a tissue mimicking phantom with no cysts was imaged 20 times for calculating the SNR. The measured SNRs for both probes are illustrated in Fig. 8. The PZT probe has a penetration depth of around $14 \mathrm{~cm}$ for single element transmissions, whereas the CMUT probe can only penetrate down to $10 \mathrm{~cm}$. However by using all the elements in transmit in the SAI sequence with transmit $f_{\#}=-1$, the CMUT probe has a penetration depth of around $14 \mathrm{~cm}$, whereas the PZT can penetrate down to almost $25 \mathrm{~cm}$. Using a SAI sequence with transmit $f_{\#}=1$ by placing the virtual sources in front of the array has increased the penetration depth for the CMUT probe down to $15 \mathrm{~cm}$, whereas the PZT can penetrate down to almost $30 \mathrm{~cm}$.
Due to the perpendicular orientation of the transmit and the receive directions, the field-of-view of RCA arrays is limited to the forward looking region in front of the array, e.g., $26 \times 26 \mathrm{~mm}^{2}$ for these probes. Two cross-planes (azimuth and C-plane) are shown in Fig. 9 and Fig. 10 at a dynamic range of $40 \mathrm{~dB}$ from a volume of $26 \times 26 \times 80 \mathrm{~mm}^{3}$ acquired with both probes using a cyst phantom and beamformed with the DAS and SMF beamforming methods. The origin corresponds to the center of the transducer surface. Data were acquired with both probes using the single element emission SAI sequence as well as the SAI sequence with a transmit $f_{\#}=-1$. The hollow cysts are located along a $10^{\circ}$ tilted plane and therefore, the lower hollow cysts are not completely visible at regions farther from the array. See the videos in the supplementary materials for the full sized DAS beamformed volumes of the cyst phantom using the SAI sequence with transmit $f_{\#}=-1$. The CNR measure is calculated in a cylindrical region centered at each of the large hollow cysts with a diameter of $8 \mathrm{~mm}$. The 


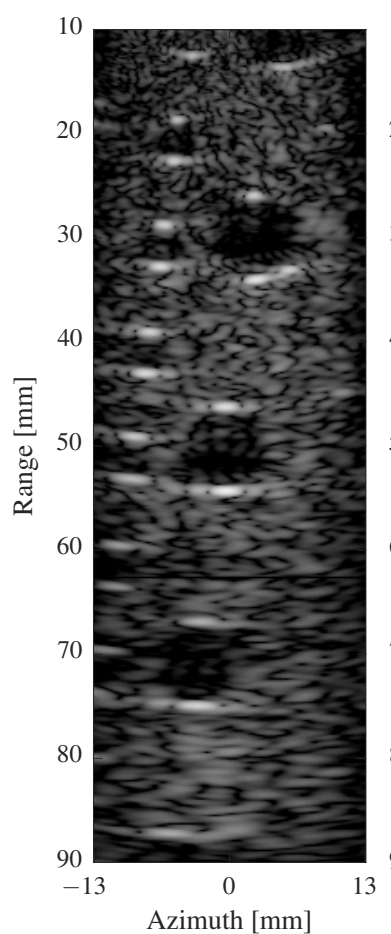

(a) PZT single elem.

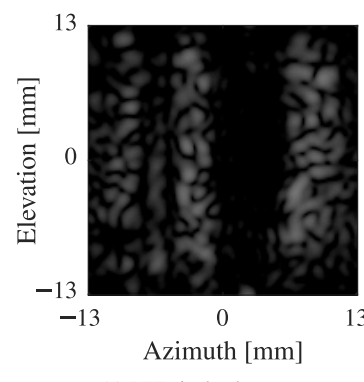

(e) PZT single elem.

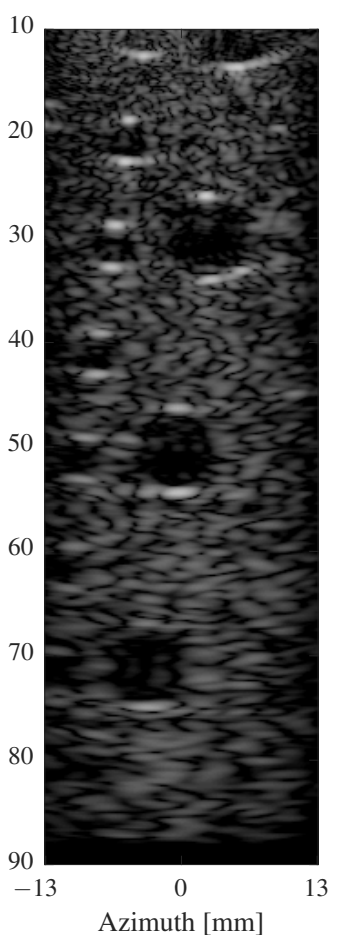

(b) PZT $f_{\#}=-1$

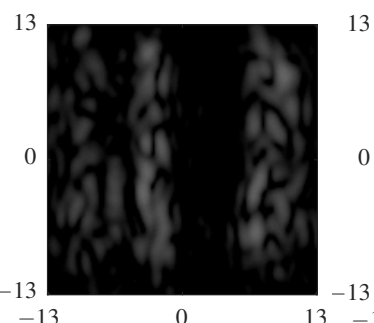

Azimuth [mm]

(f) PZT $f_{\#}=-1$

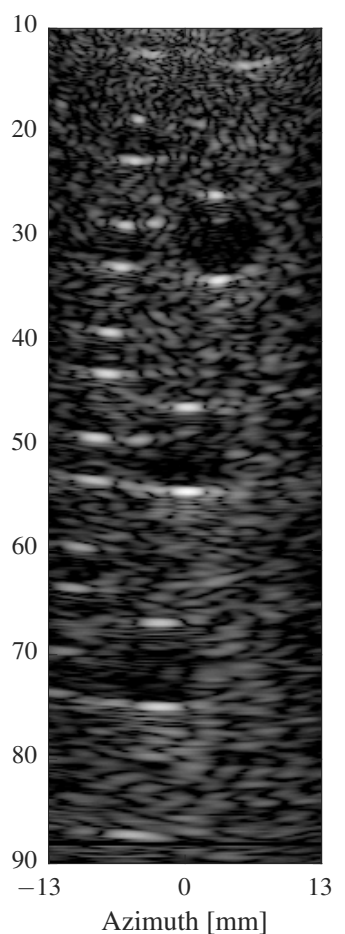

(c) CMUT single elem.

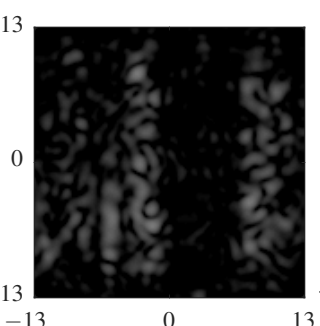

Azimuth [mm]

(g) CMUT single elem.

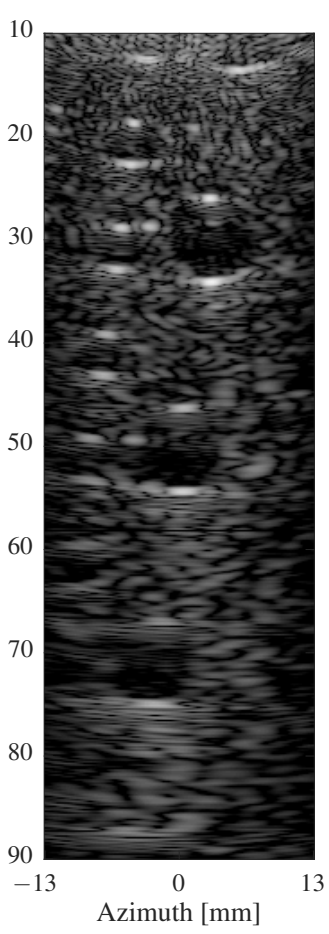

(d) CMUT $f_{\sharp}=-1$

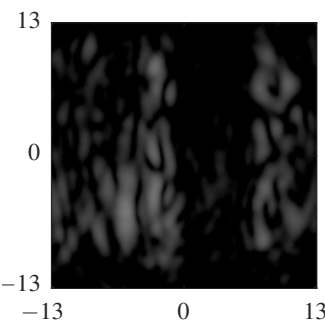

Azimuth [mm]

(h) CMUT $f_{\#}=-1$

Fig. 10. Volumetric imaging of a tissue mimicking phantom using both PZT and CMUT probes and beamformed with the SMF method. Two cross-planes (azimuth and C-plane) are shown from a volume of $26 \times 26 \times 85 \mathrm{~mm}^{3}$ at a dynamic range of $40 \mathrm{~dB}$. Data were acquired with the PZT probe using the SAI sequence with 62 single element emissions (PZT: (a) and (e)), (CMUT: (c) and (g)), and also using the SAI sequence with transmit $f_{\#}=-1$ (PZT: (b) and (f)), (CMUT: (d) and (h)). The C-planes are at the depth of $30 \mathrm{~mm}$.

calculated CNR values for each imaging sequence with each probe are shown in the Fig. 11. Due to the higher generated pressure with the PZT probe compared with the CMUT probe, the CNR of the PZT probe is almost 2 times larger than the CMUT probe.

\section{Intensity AND TEMPERATURE MEASUREMENT}

Before any in vivo measurements, the imaging sequence has to fulfill all the requirements regarding the intensity levels and safety limits as regulated by the the FDA [40] for the mechanical index $(M I \leq 1.9)$ and the derated spatial-peaktemporal-average intensity $\left(I_{\text {spta }} \leq 720 \mathrm{~mW} / \mathrm{cm}^{2}\right.$ for peripheral vessels $I_{\text {spta }} \leq 430 \mathrm{~mW} / \mathrm{cm}^{2}$ for cardiac).

The mechanical index (MI) and $\mathrm{I}_{\text {spta }}$ are measured for the synthetic aperture imaging (SAI) sequence with a transmit $f_{\#}=$ -1 at a pulse repetition frequency of $5 \mathrm{kHz}$, since it uses all row elements in transmit and thereby has the largest emitted energy. For the PZT probe they are $\mathrm{MI}=0.67$ and $\mathrm{I}_{\text {spta }}=0.53 \mathrm{~mW} / \mathrm{cm}^{2}$.

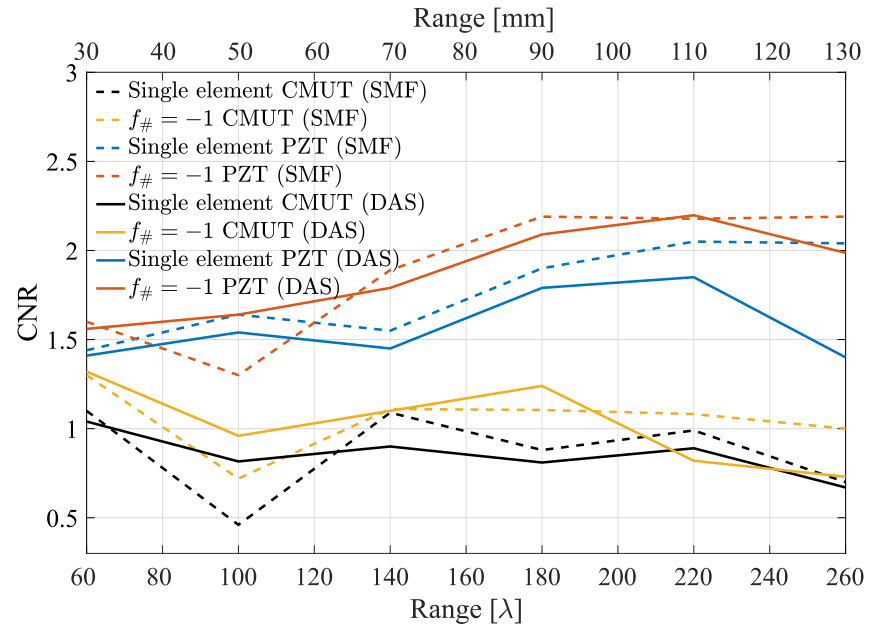

Fig. 11. CNR values measured with both PZT and CMUT probes as a function of depth. Calculated over the beamformed volumes of the cyst phantom beamformed with DAS and SMF methods. $\lambda$ for soft tissue is $0.5 \mathrm{~mm}$. 
They are MI $=0.06$ and $\mathrm{I}_{\text {spta }}=0.18 \mathrm{~mW} / \mathrm{cm}^{2}$ of the CMUT probe. All are within the FDA safety limits of $\mathrm{MI} \leq 1.9$ and $\mathrm{I}_{\text {spta }} \leq 720 \mathrm{~mW} / \mathrm{cm}^{2}$ for abdominal imaging [40]-[42]. For the SAI sequence with a transmit $f_{\#}=1$ at a pulse repetition frequency of $5 \mathrm{kHz}$, the measured $\mathrm{MI}=0.88$ and $\mathrm{I}_{\text {spta }}=5.5 \mathrm{~mW} / \mathrm{cm}^{2}$ for the PZT probe, and MI $=0.13$ and $\mathrm{I}_{\text {spta }}=0.55 \mathrm{~mW} / \mathrm{cm}^{2}$ for the CMUT probe are also both within the FDA safety limits. Therefore, both sequences can be scaled to a larger excitation voltage for the in vivo measurements before reaching the FDA limits, thus achieving a higher penetration depth. All sequences are MI limited, and the transmit voltage could be scaled by a factor between 2.16 and 31.7 giving an increased SNR of 6.7 to $30 \mathrm{~dB}$. This could result in a penetration increase of $1.1 \mathrm{~cm}$ to $5 \mathrm{~cm}$.

Another criteria that has to be addressed is the heating of the probe, which has to be within the FDA safety limits [40]. The linear voltage regulators used for the amplifiers in the prototype probes are dissipating power and generating waste heat. Therefore, the temperature rise in the probe is a combination of heating produced by the linear regulators as well as the transducer arrays. To separate the heating caused by the amplifiers in the handle from the transducers themselves, two spots on the probes were measured for temperature changes in a still air environment. One sensor located at the sole of the probe and the other sensor was located on the body of the probe, where the amplifiers are located. The temperature rise of both probes is dominated by the dissipated heat of the linear voltage regulators. However, the absolute temperature of both probes in still air does not reach the human body temperature. Both probes satisfy the FDA safety limits on the absolute temperature in still air, which requires the absolute temperature to be less than human body temperature. However, on the temperature rise, due to the heat generated by the linear voltage regulators, both probes did not satisfy the requirement of less than $10^{\circ} \mathrm{C}$ temperature rise in 60 minutes and therefore for future clinical use, modifications to the power supplies of these prototype probes need to be made.

\section{DISCUSSION}

It was shown that Row-column arrays theoretically can attain a larger resolution than fully populated arrays, when the element count is larger than 5, when taking side-lobes into account. The scaling of the array size and its attainable resolution is proportional to $N$ - the number of elements on the side of the array. For a fully populated array it is proportional to $N^{2}$, making it difficult, if not impossible, to attain a high resolution at large depths. Using two $62+62$ element arrays it was shown that synthetic aperture imaging and two beamforming schemes for both simulations and measurements attain the theoretical resolution with depth. The best lateral resolution was achieved with the single element transmissions SAI sequence using DAS. In Fig. 7a and Fig. 7b by using SMF, the CR values have been improved for both CMUT and PZT probes for the single element transmission SAI sequence. However, for the CMUT probe, using the defocused SAI sequence, no significant difference between DAS and SMF has been observed. For the PZT probe, on the other hand, using SMF for the defocused
SAI sequence has worsened the CR compared to DAS. This might be improved by having a more precise model of the arrays. For both probes the seventh wire at the depth of $154 \lambda$ had a lower main-lobe to side-lobe ratio for compared SMF method to DAS, thus increased the CR value at that depth.

The arrays were in simulations compared to a fully populated array, with the same dimensions. It theoretically attained a $34 \%$ better resolution for all metrics, but it should be kept in mind that it must employ 3,844 connections to the transducer, and that 1024 emissions were used for imaging rather than the 62 emissions for row-column arrays. The $34 \%$ improved resolution, thus, comes at the price of 31 times more connections and a 16 times lower volume frame rate.

It was also demonstrated that the penetration depth for the $3 \mathrm{MHz}$ row-column arrays can be up to $30 \mathrm{~cm}$ in phantom measurements with an attenuation of $0.5 \mathrm{~dB} /[\mathrm{MHz} \mathrm{cm}]$. This can be attested to the large size of the arrays, which scales with $N^{2}$. The overall sensitivity of the CMUT array was lower than the PZT probe, primarily due to the lower pressure emitted by the probe. This limited the penetration depth to between 10-15 $\mathrm{cm}$ depending on the SA imaging scheme. The CMUT probe, however, had a larger bandwidth and therefore a higher axial resolution. It is also more flat than the PZT probe, which had a slight curvature, probably due to the mounting [23].

Using single elements for transmission, the CMUT probe has a lower penetration depth on a tissue mimicking phantom compared to the PZT probe. On the contrary by using all the elements in transmit, both probes penetrate down to $15 \mathrm{~cm}$, and the PZT probe even down to $30 \mathrm{~cm}$. In the same way, the CNR values increased, when using all the elements in transmit. Placing the virtual focus lines in front of the transducer would increase the penetration depth further, but larger transmit $f_{\#}$ values will degrade the spatial resolution. For SAI sequences with the transmit focus in front of the array, the transmitted acoustic energy is focused along a line in contrast to a point by using fully addressed 2-D arrays, and therefore for the same size of the 2-D arrays, the MI and the $\mathrm{I}_{\text {spta }}$ are lower for RCA 2-D arrays.

The cyst phantom measurements also yielded volumetric images at a possible volume rate of $135 \mathrm{~Hz}$ down to $9 \mathrm{~cm}$. The volumes clearly showed the cysts in the phantom for both beamforming schemes, with a better contrast for the spatial matched scheme at the price of a worse axial resolution. The SMF approach is also considerably more computationally intense.

Theoretically, transmitting with row elements and receiving with column elements should image exactly the same rectilinear volume as transmitting with column elements and receiving with row elements. However, because of the manufacturing process, the sensitivity of row and column elements might be slightly different. For the PZT probe the difference is small, since transmitting with row elements and receiving the echoes with column elements or vice versa, are similar as shown in Fig. 12. For the CMUT probe, because of a capacitive substrate coupling of the bottom electrodes, as discussed in [23], the receive sensitivity of the bottom elements is lower and therefore in our imaging set-up, the elements with higher receive sensitivity, i.e., the top elements, are chosen for 


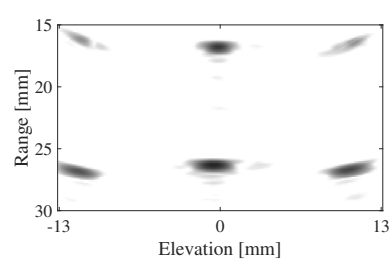

(a)

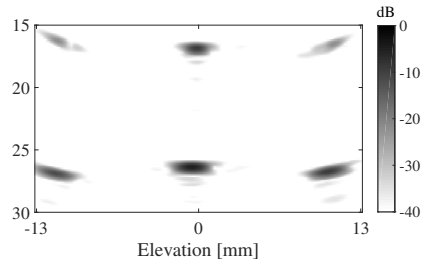

(b)
Fig. 12. Two azimuth planes from volumes of $26 \times 26 \times 15 \mathrm{~mm}^{3}$ acquired with the PZT probe using single element transmission SAI sequence at a dynamic range of $40 \mathrm{~dB}$ are shown when: (a) row elements transmit and column elements receive, and (b) column elements transmit and row elements receive.

receiving, while the bottom elements are used for transmitting.

A drawback of the row-column array is that only a rectilinear volume is investigated, so only the volume beneath the transducer area can be beamformed due to the rapid fall in energy outside the area. This can be mitigated by employing a lens on the array to both spread out the energy in transmit and make collection of energy outside the rectilinear region possible in receive. Such arrays have been investigated in [43] with lenses in front of the arrays investigated in this paper.

The SAI technique is based on coherently summing the overlapping beams acquired from subsequent transmit events, and therefore it is vulnerable to motion in the imaged medium. In 2-D imaging, in plane motion can be compensated by correlating the low- and high-resolution images in order to correct for the motion artifacts. It has previously been shown that motion compensation in 2-D SAI can be used for fully compensating the reduction in contrast and resolution [44]. It has also been shown that row-column arrays can be used for finding the full 3-D velocity vector [45], [46], and these techniques can be combined to employ motion compensation in 3-D.

\section{CONCLUSION}

In this paper, the imaging performance of two prototyped 62+62 RCA 2-D array probes fabricated in CMUT and PZT transducer technologies were demonstrated quantitatively and comparatively. Using SAI technique both probes were able to image down to $14 \mathrm{~cm}$ at a volume rate of $88 \mathrm{~Hz}$. DAS and SMF beamforming methods were both able to perform dynamic transmit-receive focusing throughout the rectilinear field-of-view. The performance of both probes was evaluated through simulation and experiments. Results show that both probes can image a rectilinear volume in front of the transducer successfully. Integrated hardware apodization along each line-element effectively removed the ghost echoes without altering the main echo's beam width. It was demonstrated that volumetric imaging with equipment in the price range of conventional 2-D imaging is possible. Both probes were prototypes and not optimized, which limited the imaging performance. Future work will focus on configuring the probes for better performance through adjusting the DC bias voltage for the CMUT probe for achieving higher penetration and using a better shielding method for both probes to eliminate the reflections within the probes.
The results of this study have demonstrated the promising potentials of RCA 2-D arrays compared with fully addressed 2-D arrays, which are their low channel count, low MI and $\mathrm{I}_{\text {spta }}$ values, and high penetration depth. It was shown that, due to one-way focusing of RCA 2-D arrays in each lateral dimension, the spatial resolution is lower than fully addressed arrays, however that can be compensated by increasing the size of the array by $36 \%$ in each lateral dimension or $125 \%$ when taking apodization for side-lobe reduction into account. Moreover, the contrast resolution was improved by using SMF beamforming method.

\section{ACKNOWLEDGMENT}

This work was financially supported by grant 82-2014-4 from the Danish National Advanced Technology Foundation and from BK Ultrasound ApS, Herlev, Denmark.

\section{BIBLIOGRAPHIES}

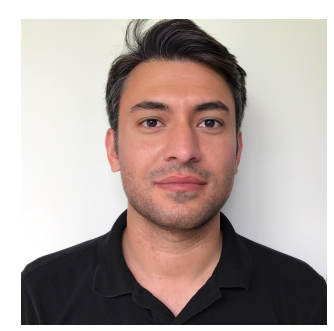

Hamed Bouzari (S'14-M'16) received the B.Sc. degree in electrical engineering from Mazandaran University, Babolsar, Iran, in 2006, the M.Sc. degree in electrical engineering from Zanjan University, Zanjan, Iran, in 2009, and the Ph.D. degree in biomedical engineering from the Technical University of Denmark, Kongens Lyngby, Denmark, in 2016. He was a Researcher with the Austrian Academy of Sciences, Vienna, Austria, from 2010 to 2013. In 2017, he worked as Post-Doctoral Researcher with the Center for Fast Ultrasound Imaging, Technical University of Denmark. He is currently a Senior Researcher at Fingerprint Cards AB, Herlev, Denmark. His current research interests include in-display ultrasonic fingerprint imaging for mobile phones.

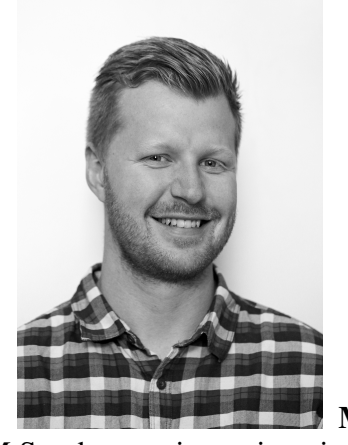
Technical University of Denmark, Kgs. Lyngby, Denmark, in 2012 and 2015, respectively. He received his Ph.D. degree in 2018 also from the Technical University of Denmark with focus on micromachined ultrasonic transducers for three-dimensional imaging. He is currently a postdoctoral researcher at the Technical University of Denmark. His research interests include all facets of ultrasonic imaging transducers. 


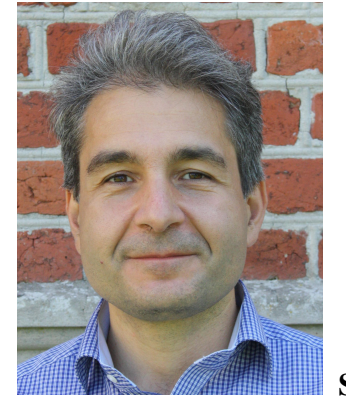
of Science in electrical engineering in 1996 from the Technical University - Sofia (TU - Sofia), and a Ph.D. degree in 2001 fromt the Technical University of Denmark (DTU). From 2001 till 2009 he was an Associate Professor in Electronics and Signal Processing at DTU. Svetoslav Nikolov is Senior IEEE Member since 2011 for his pioneering work in the field of synthetic aperture imaging and flow estimation. In 2008, he joined BK Medical Aps to work on the commercial implementation of the technologies developed at DTU. $\mathrm{He}$ is an Analogic Fellow since 2015 as a recognition for his work in research and development.

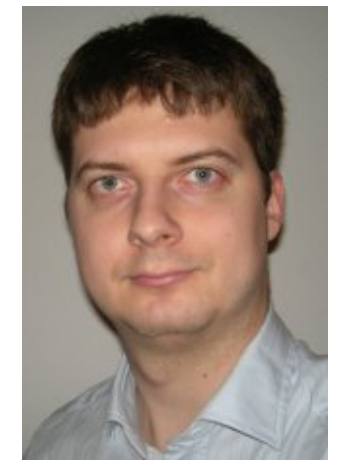

Mathias Bo Stuart received the M.Sc. and $\mathrm{Ph} . \mathrm{D}$. degrees in Computer Engineering from the Technical University of Denmark, Lyngby, Denmark in 2006 and 2010 respectively. He is currently an Associate Professor with the Biomedical Engineering Section, Department of Health Technology at the Technical University of Denmark. His research interests include synthetic aperture methods for both anatomical and flow imaging in both 2-D and 3-D, ultrasound systems, and real-time implementations of ultrasound processing algorithms.

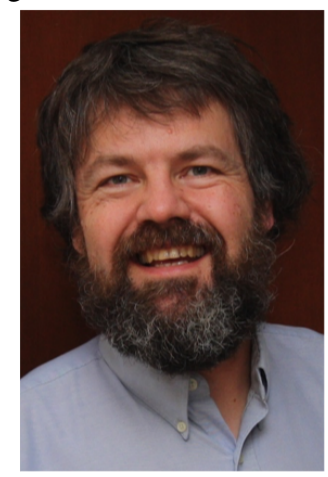

Erik Vilain Thomsen was born in Aarhus, Denmark, in 1964. He received the M.Sc. degree in physics from the University of Southern Denmark, Odense, Denmark, in 1992 and the Ph.D. degree in electrical engineering from the Technical University of Denmark (DTU), Kongens Lyngby, Denmark, in 1998. $\mathrm{He}$ is currently Professor at the Department of Health Technology, Technical University of Denmark (DTU) where he is also the Head of the MEMS Applied Sensors Group and Head of Division with responsibility for the educations in healthcare engineering. He teaches classes in solid state electronics, microtechnology, and nano and microfabrication. His current research interests include all aspects of capacitive micromachined ultrasonic transducers (CMUTs), general MEMS technology, and piezoelectric MEMS. Dr. Thomsen received the Danish National Advanced Technology Foundation - "Grand
Solution Prize" in 2017, the AEG Electron Prize in 1995, and has received several teaching awards at DTU.

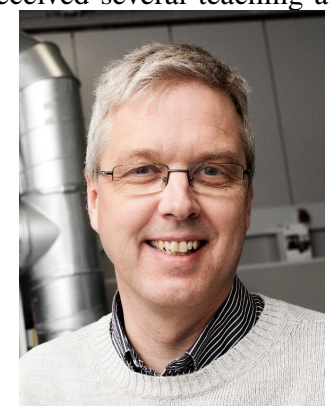

Jørgen Arendt Jensen (M'93-SM'02F'12) received the MSc degree in 1985, the Ph.D. degree in 1989, and the Dr.Techn. degree all from the university in 1996. Since 1993, he has been a Full Professor of Biomedical Signal Processing with the Department of Electrical Engineering, Technical University of Denmark. He has been the founder and head of the Center for Fast Ultrasound Imaging since its inauguration in 1998. CFU has contributed with innovations in transverse oscillation vector flow imaging, synthetic aperture flow imaging in $2 \mathrm{D}$ and $3 \mathrm{D}$, ultrasound simulation, research scanners, and row-column probes and beamforming. He has published more than 450 journal and conference papers on signal processing and medical ultrasound and the book Estimation of Blood Velocities Using Ultrasound (Cambridge Univ. Press), 1996. He is also the developer and maintainer of the Field II simulation program. He has been a visiting scientist at Duke University, Stanford University, and the University of Illinois at Urbana-Champaign. He was founder and head of the Biomedical Engineering group from 2007 to 2010. In 2003, he was one of the founders of the biomedical engineering program in Medicine and Technology, which is a joint degree program between the Technical University of Denmark and the Faculty of Health and Medical Sciences at the University of Copenhagen. The degree is one of the most sought-after engineering degrees in Denmark. He was chairman of the study board from 2003 to 2010 and Adjunct Professor with the University of Copenhagen from 2005 to 2010 . He has given a number of short courses on simulation, synthetic aperture imaging, and flow estimation at international scientific conferences and teaches biomedical signal processing and medical imaging at the Technical University of Denmark. His research is centered around simulation of ultrasound imaging, synthetic aperture imaging, vector blood flow estimation, 3D imaging, row-column probes, and construction of ultrasound research systems. Dr. Jensen has given more than 60 invited talks at international meetings and received several awards for his research, most recently the Grand Solutions Prize from the Danish Minister of Science and the order of the Dannebrog by her Majesty the Queen of Denmark.

\section{REFERENCES}

[1] O. T. von Ramm, S. W. Smith, and H. G. Pavy, "High speed ultrasound volumetric imaging system - Part II: Parallel processing and image display," IEEE Trans. Ultrason., Ferroelec., Freq. Contr., vol. 38, pp. 109-115, 1991.

[2] D. H. Turnbull and F. S. Foster, "Beam steering with pulsed twodimensional transducer arrays," IEEE Trans. Ultrason., Ferroelec., Freq. Contr., vol. 38, no. 4, pp. 320-333, July 1991.

[3] S. W. Smith, H. G. Pavy, and O. T. von Ramm, "High speed ultrasound volumetric imaging system - Part I: Transducer design and beam steering," IEEE Trans. Ultrason., Ferroelec., Freq. Contr., vol. 38, pp. 100-108, 1991.

[4] C. E. Morton and G. R. Lockwood, "Theoretical assessment of a crossed electrode 2-D array for 3-D imaging," in Proc. IEEE Ultrason. Symp., 2003, pp. 968-971.

[5] M. F. Rasmussen and J. A. Jensen, "3D ultrasound imaging performance of a row-column addressed 2D array transducer: a simulation study," in Proc. SPIE Med. Imag., 2013, pp. 1-11, 86750C.

[6] _ " "3-D ultrasound imaging performance of a row-column addressed 2-D array transducer: A measurement study," in Proc. IEEE Ultrason. Symp., July 2013, pp. 1460-1463. 
[7] C. E. M. Démoré, A. Joyce, K. Wall, and G. Lockwood, "Real-time volume imaging using a crossed electrode array," IEEE Trans. Ultrason. Ferroelec., Freq. Contr., vol. 56, no. 6, pp. 1252-1261, 2009.

[8] A. W. Joyce and G. R. Lockwood, "Variably polarized ceramic for passive aperture apodization," in Proc. IEEE Ultrason. Symp., 2012, pp. 1557-1559.

[9] T. L. Christiansen, M. F. Rasmussen, J. A. Jensen, and E. V. Thomsen, "Row-column addressed 2-D CMUT arrays with integrated apodization," in Proc. IEEE Ultrason. Symp., 2014, pp. 600-603.

[10] N. M. Daher and J. T. Yen, "2-D array for 3-D ultrasound imaging using synthetic aperture techniques," IEEE Trans. Ultrason., Ferroelec., Freq. Contr., vol. 53, no. 5, pp. 912-924, 2006.

[11] M. F. Rasmussen, T. L. Christiansen, E. V. Thomsen, and J. A Jensen, "3-D imaging using row-column-addressed arrays with integrated apodization - Part I: Apodization design and line element beamforming," IEEE Trans. Ultrason., Ferroelec., Freq. Contr., vol. 62, no. 5, pp. 947 958,2015

[12] C. Ceroici, T. Harrison, and R. Zemp, "Fast orthogonal row-column electronic scanning (forces) with top orthogonal to bottom electrode (tobe) arrays," IEEE Trans. Ultrason., Ferroelec., Freq. Contr., vol. 64 no. 6, pp. 1009-1014, 2017.

[13] A. S. Logan, L. L. P. Wong, A. I. H. Chen, and J. T. W. Yeow, "A 32 x 32 element row-column addressed capacitive micromachined ultrasonic transducer," IEEE Trans. Ultrason., Ferroelec., Freq. Contr., vol. 58, no. 6, pp. 1266-1271, June 2011.

[14] R. K. W. Chee, A. Sampaleanu, D. Rishi, and R. J. Zemp, “Top orthogonal to bottom electrode (TOBE) 2-D CMUT arrays for 3-D photoacoustic imaging," IEEE Trans. Ultrason., Ferroelec., Freq. Contr., vol. 61, no. 8 , pp. 1393-1395, 2014.

[15] A. Sampaleanu, P. Zhang, A. Kshirsagar, W. Moussa, and R. Zemp, "Toporthogonal-to-bottom-electrode (TOBE) CMUT arrays for 3-D ultrasound imaging." IEEE Trans. Ultrason., Ferroelec., Freq. Contr., vol. 61, no. 2 , pp. 266-276, 2014.

[16] R. K. W. Chee and R. J. Zemp, "Feasibility of modulation-encoded TOBE CMUTs for real-time 3-D imaging," IEEE Trans. Ultrason., Ferroelec. Freq. Contr., vol. 62, no. 4, pp. 771-775, 2015.

[17] T. L. Christiansen, M. F. Rasmussen, J. P. Bagge, L. N. Moesner, J. A Jensen, and E. V. Thomsen, "3-D imaging using row-column-addressed arrays with integrated apodization — part II: Transducer fabrication and experimental results," IEEE Trans. Ultrason., Ferroelec., Freq. Contr., vol. 62, no. 5, pp. 959-971, 2015.

[18] C. H. Seo and J. T. Yen, "A 256 x 256 2-D array transducer with rowcolumn addressing for 3-D rectilinear imaging," IEEE Trans. Ultrason., Ferroelec., Freq. Contr., vol. 56, no. 4, pp. 837-847, April 2009.

[19] J. T. Yen, C. H. Seo, S. I. Awad, and J. S. Jeong, "A dual-layer transducer array for 3-D rectilinear imaging," IEEE Trans. Ultrason., Ferroelec. Freq. Contr., vol. 56, no. 1, pp. 204-212, 2009.

[20] K. Latham, C. Ceroici, C. A. Samson, R. J. Zemp, and J. A. Brown, "Simultaneous azimuth and fresnel elevation compounding: A fast 3-d imaging technique for crossed-electrode arrays," IEEE Trans. Ultrason. Ferroelec., Freq. Contr., vol. 65, no. 9, pp. 1657-1668, 2018.

[21] M. Flesch, M. Pernot, J. Provost, G. Ferin, A. Nguyen-Dinh, M. Tanter, and T. Deffieux, "4D in vivo ultrafast ultrasound imaging using a rowcolumn addressed matrix and coherently-compounded orthogonal plane waves," Phys. Med. Biol., vol. 62, pp. 4571-4588, 2017.

22] J. A. Jensen, H. Holten-Lund, R. T. Nilsson, M. Hansen, U. D. Larsen, R. P. Domsten, B. G. Tomov, M. B. Stuart, S. I. Nikolov, M. J. Pihl, Y. Du, J. H. Rasmussen, and M. F. Rasmussen, "SARUS: A synthetic aperture real-time ultrasound system," IEEE Trans. Ultrason., Ferroelec. Freq. Contr., vol. 60, no. 9, pp. 1838-1852, 2013.

[23] M. Engholm, H. Bouzari, T. L. Christiansen, C. Beers, J. P. Bagge, L. N. Moesner, S. E. Diederichsen, M. B. Stuart, J. A. Jensen, and E. V. Thomsen, "Probe development of CMUT and PZT row-columnaddressed 2-D arrays," Sens. Actuators A: Phys., vol. 273, pp. 121-133, 2018.

[24] J. A. Jensen and P. Gori, "Spatial filters for focusing ultrasound images," in Proc. IEEE Ultrason. Symp., 2001, pp. 1507-1511.

[25] K. S. Kim, J. Liu, and M. F. Insana, "Efficient array beam forming by spatial filtering for ultrasound B-mode imaging," J. Acoust. Soc. Am. vol. 120 , no. 2 , pp. 852-861, 2006.

[26] J. T. Yen, "Beamforming of sound from two-dimensional arrays using spatial matched filters," J. Acoust. Soc. Am., vol. 134, no. 5, pp. $3697-$ 3704, 2013.

[27] J. A. Jensen, S. Nikolov, K. L. Gammelmark, and M. H. Pedersen, "Synthetic aperture ultrasound imaging," Ultrasonics, vol. 44, pp. e5-e15, 2006.
[28] M. Engholm, T. L. Christiansen, C. Beers, J. P. Bagge, L. N. Moesner, H. Bouzari, A. Lei, M. Berkheimer, M. B. Stuart, J. A. Jensen, and E. V. Thomsen, "A hand-held row-column addressed CMUT probe with integrated electronics for volumetric imaging," in Proc. IEEE Ultrason. Symp., 2015, pp. 1-4.

[29] H. Bouzari, M. Engholm, T. L. Christiansen, M. B. Stuart, S. I. Nikolov, E. V. Thomsen, and J. A. Jensen, "Volumetric ultrasound imaging with row-column addressed 2-D arrays using spatial matched filter beamforming," in Proc. IEEE Ultrason. Symp. IEEE, 2015, pp. 1-

[30] H. Bouzari, M. Engholm, T. L. Christiansen, C. Beers, A. Lei, M. B Stuart, S. I. Nikolov, E. V. Thomsen, and J. A. Jensen, "Volumetric synthetic aperture imaging with a piezoelectric 2-D row-column probe," in Proc. SPIE Med. Imag., vol. 9790, 2016, pp. 1-9.

[31] T. L. Szabo, Diagnostic ultrasound imaging: Inside out, 2nd ed. Elsevier (Oxford, UK), 2014.

[32] M. Karaman, I. O. Wygant, O. Oralkan, and B. T. Khuri-Yakub, "Minimally redundant 2-D array designs for 3-D medical ultrasound imaging," IEEE Trans. Med. Imag., vol. 7, pp. 1051-1061, jul 2009.

[33] K. S. Kim and T. K. Song, "High volume rate 3-D ultrasound imaging using cross array based on synthetic transmit focusing," in Proc. IEEE Ultrason. Symp., vol. 2, aug 2004, pp. 1409-1412.

[34] J. A. Jensen and N. B. Svendsen, "Calculation of pressure fields from arbitrarily shaped, apodized, and excited ultrasound transducers," IEEE Trans. Ultrason., Ferroelec., Freq. Contr., vol. 39, pp. 262-267, 1992.

[35] J. A. Jensen, "Field: A program for simulating ultrasound systems," Med. Biol. Eng. Comp., vol. 10th Nordic-Baltic Conference on Biomedical Imaging, Vol. 4, Supplement 1, Part 1, pp. 351-353, 1996.

[36] _ - "A multi-threaded version of Field II," in Proc. IEEE Ultrason. Symp. IEEE, 2014, pp. 2229-2232.

[37] D. Vilkomerson, J. Greenleaf, and V. Dutt, "Towards a Resolution Metric for Medical Ultrasound Imaging," in Proc. IEEE Ultrason. Symp., 1995, pp. 1405-1410.

[38] K. Ranganathan and W. F. Walker, "Cystic resolution: A performance metric for ultrasound imaging systems," IEEE Trans. Ultrason., Ferroelec. Freq. Contr., vol. 54, no. 4, pp. 782-792, 2007.

[39] D. A. Guenther and W. F. Walker, "Generalized cystic resolution: metric for assessing the fundamental limits on beamformer performance,' IEEE Trans. Ultrason., Ferroelec., Freq. Contr., vol. 56, no. 1, pp. 77-90, January 2009.

[40] FDA, "Information for manufacturers seeking marketing clearance of diagnostic ultrasound systems and transducers," Center for Devices and Radiological Health, United States Food and Drug Administration, Tech. Rep., 2008.

[41] J. A. Jensen, M. F. Rasmussen, M. J. Pihl, S. Holbek, C. A. VillagomezHoyos, D. P. Bradway, M. B. Stuart, and B. G. Tomov, "Safety assessment of advanced imaging sequences, I: Measurements," IEEE Trans. Ultrason. Ferroelec., Freq. Contr., vol. 63, no. 1, pp. 110-119, 2016.

[42] J. A. Jensen, "Safety assessment of advanced imaging sequences, II: Simulations," IEEE Trans. Ultrason., Ferroelec., Freq. Contr., vol. 63 , no. 1, pp. 120-127, 2016

[43] H. Bouzari, M. Engholm, C. Beers, S. I. Nikolov, M. B. Stuart, E. V. Thomsen, and J. A. Jensen, "Curvilinear 3-d imaging using row-column addressed 2-d arrays with a diverging lens: Phantom study," IEEE Trans. Ultrason., Ferroelec., Freq. Contr., vol. 65, no. 7, pp. 1182-1192, 2018

[44] K. L. Gammelmark and J. A. Jensen, "2-D tissue motion compensation of synthetic transmit aperture images," IEEE Trans. Ultrason., Ferroelec. Freq. Contr., pp. 594-610, April 2014.

[45] S. Holbek, T. L. Christiansen, M. B. Stuart, C. Beers, E. V. Thomsen, and J. A. Jensen, "3-D vector flow estimation with row-column addressed arrays," IEEE Trans. Ultrason., Ferroelec., Freq. Contr., vol. 63, no. 11, pp. 1799-1814, 2016.

[46] S. Holbek, M. B. Stuart, and J. A. Jensen, "Volumetric 3-D vector flow measurements using a 62+62 row-column addressed array," in Proc. IEEE Ultrason. Symp., 2017, pp. 1-4. 